| Original | Article |

\title{
Assessment of urinary tract infection and their resistance to antibiotics in diabetic and non-diabetic patients
}

\author{
Nureen Zahra, Kanwal Rehman, Rana Aqeel, Asia Parveen and Muhammad Sajid Hamid Akash
}

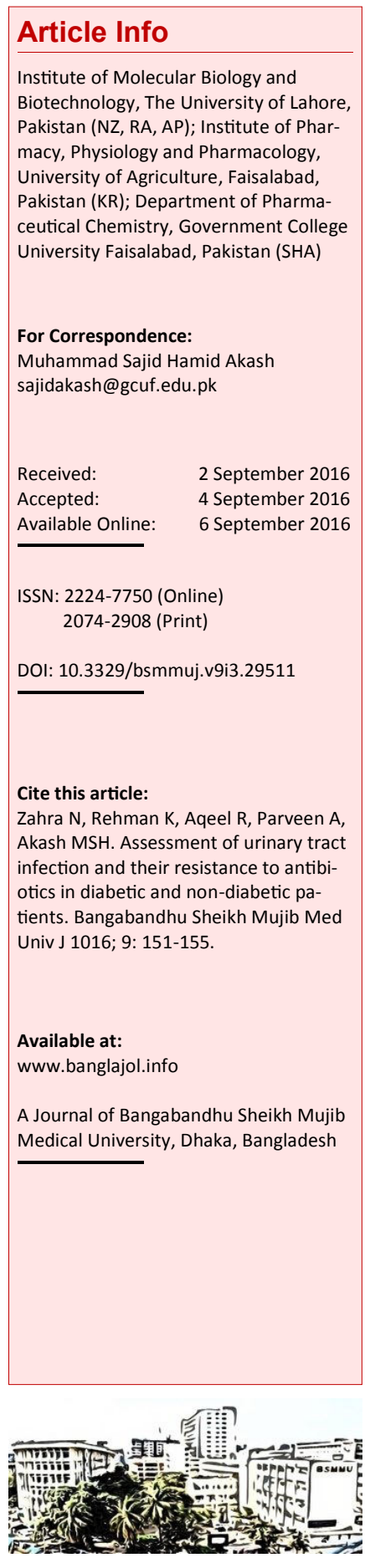

\section{Abstract}

The study was undertaken to determine the influence of diabetes mellitus on the uropathogens and antibiotic sensitivity pattern among patients with urinary tract infections (UTIs). A crosssectional study was conducted on 150 diabetic and 250 non-diabetic patients. Out of 160 uropathogenic isolates, Escherichia coli was found as a leading pathogen i.e. $46.2 \%$ followed by Candida spp. 30.6\%, Streptococcus faecalis 15.6\%, Pseudomonas aeruginosa 3.1\%, Pneumococcus. 1.2\%, mathicilline sensitive $S$. aureus $1.2 \%$, mathicilline resistant $S$. aureus $0.6 \%$, Proteus spp. $0.6 \%$ and vancomycin resistant Enterococcus spp. $0.6 \%$. The results indicated that prevalence of UTIs was significantly higher in diabetic patients than in non-diabetic subjects. E. coli was found to be the most common isolate. It was observed that UTIs in diabetic patients was more in female diabetic patients than in male patients. Investigation of bacteriuria in diabetic patients for UTIs is an important tool for the treatment and prevention of renal complications.

\section{Introduction}

Diabetes mellitus (DM) has been characterized by a metabolic disorder of multipule etiology including hyperglycemia and hyslipidemia along with disturbance in carbohydrate, fat and protein metabolism. $\underline{1-3} \mathrm{DM}$ and its associated complications are one of the most prevalent diseases worldwide. Development of multidrug resistant uropathogenic strains in associated with DM is being escalated that helps to determine the prevalence of urinary tract infections (UTIs) among diabetic patients and sensitivity of bacterial isolates against various types of antimicrobial agents. UTIs is one of the most common diseases that encounters in clinical practice today. 4 It is particularly common infection in diabetic patients that occurs in the all ages of both males and females $\underline{5}$ and if it is left untreated, causes considerable morbidity. $.6,7$

UTIs are mainly originated by the bacterial species. Escherichia coli belongs to the specific serogroups of uropathogenic and is considered as frequently identified organism. Serogroups have many virulence factors that are specific for invasion of urinary epithelium. 8 E. coli is the most common cause of the uncomplicated UTI and account for about $95 \%$ of all infections. $9 E$. coli is the main causative factor for the induction of UTIs in women and also increases the likelihood of persistent UTIs.

DM has a number of effects on genitourinary system and has long been considered to be a predisposing factor for UTIs. A characteristic feature observed in UTIs in diabetic patients in the presence of asymptomatic bacteriuria, is more in female than in male patients. The exact reason is not clear, but may be attributed to a number of factors. These include impairment of granulocyte function, increased adherence of uropathogens to uroepithelial cells, dysfunctional bladder and increased in sugar content of urine. 10 Meiland et al. found that longer duration of DM was associated with the risk of asymptomatic bacteriuria.11 The prevalence of asymptomatic bacteriuria is 15 to $30 \%$ higher in diabetic than in non-diabetic women.

To the best of our knowledge, limited data regarding the correlation of UTIs and their susceptibility to antibacterial agents in diabetic and non-diabetic patients is available online. The aim of present study was the assessment of UTIs and their susceptibility to various antibacterial agents among diabetic and nondiabetic agents in one of the most populated cities i.e. Lahore of Punjab province, in Pakistan.

\section{Materials and Methods}

\section{Study design}

A cross-sectional study was carried out at one of the largest government hospital located in 
Lahore, Punjab province, in Pakistan and experimental analysis was conducted at department of microbiology, Institute of Molecular Biology and Biotechnology, The University of Lahore. Consecutive diabetic and non-diabetic patients of any sex who visited the hospital, were approached to participate in this study. A total of 400 urine samples from the outdoor and indoor patients were collected for specimen culturing. According to the clean-catch procedure, midstream urine samples were collected using sterile container on the same day of enrolment. After collection, urine samples were immediately brought to the microbiology laboratory for further analysis. In this study, the exclusion criteria included the chronic renal disease, known underlying renal pathology, use of antimicrobial therapy during the last month and pregnancy. Informed written consent was also obtained from individual participant and data regarding the clinical characteristics were collected on pre-tested questionnaire.

\section{Bacterial isolation}

Urinalysis was performed for all urine samples. Centrifuged urine was taken in a dropper and put a drop on clean slide for microscopy. By using standard quantitative loop, $0.001 \mathrm{~mL}$ was used to inoculate urine samples on cystein lactose electrolyte deficient Agar and MacConkey's agar plates and incubated at $37^{\circ} \mathrm{C}$ for 24 hours. When at least $10^{5}$ colony forming unit (CFU)/mL of urine was present, UTI was considered to be occurred. After incubation, we examined each plate for etiological agent and colony count. We also isolated different colonies present on the culture plate and performed further tests for bacterial identification.

\section{Bacterial identification}

We identified the bacteria isolated with the help of Gram's stain, rapid tests (catalase, oxidase, coagulase) using API-20E test kit.

\section{Determination of antimicrobial susceptibility}

We determined the antimicrobial susceptibility of bacteriuria using disc diffusion method. Nutrient agar (Merk, Germany) at the rate of $14.5 \mathrm{~g} /$ liter was prepared. Antibiotic susceptibility test was performed by Kirby Bauer modified disc diffusion method. Following antibiotic discs were used against bacterial pathogens: ampicillin, amoxicillin, clavulanic acid, cefepime, cefoperazone, imipenem, meropenem, vancomycin, amikacin, gentamycin, doxycycline, ciprofloxacin, levofloxacin, sulfamethoxazole, nitrofurantion, pipemedic acid and nalidixic acid.

We standardized the turbidity of test inoculums using McFarland nephlometer tube (\# 0.5). As the inoculum was prepared in Mueller Hinton broth, in order to estimate bacterial cell density, we prepared $1 \%(\mathrm{v} / \mathrm{v})$ sulfuric acid in Mueller Hinton broth and
$1.175 \%$ aqueous solution of barium chloride. Standard solution of turbidity was prepared by adding $0.05 \mathrm{~mL} 1.175 \%$ barium chloride solution in $9.9 \mathrm{~mL} \mathrm{1 \%}$ sulfuric acid. A loop full from colony was taken and transferred to $5 \mathrm{~mL}$ of Mueller Hinton broth and broth was incubated at $37^{\circ} \mathrm{C}$ for 24 hours. To get appropriate cell density $\left(150 \times 10^{6}\right.$ $\mathrm{CFU} / \mathrm{mL}$ ), we compared the turbidity with 0.5 McFarland standardized nephlometer tube and standardized inoculum suspension was inoculated within 15-20 min. Antibiotic discs released impregnated antibiotic into the surrounding medium when placed on the plates containing uniformly inoculated and actively growing microorganisms. The plates were inverted and placed in an incubator at $37^{\circ} \mathrm{C}$.

\section{Interpretation of inhibition zones}

Results were reported either as sensitive (S), resistant (R) and intermediate (I) according to the interpretation table supplied by the company (Oxoid limited, England)

\section{Results \\ Correlation of UTIs among diabetic and non- diabetic patients}

A total 160 patients of UTIs have been studied, out of which 80 were diabetic and 80 were non-diabetic. Out of 160 patients, 69 were males and 91 were females, which shows that more number of females suffered from UTIs than males. Out of 80 diabetic patients, 29 were males and 51 were females. Whereas, out of 80 non-diabetic patients, 40 were males and 40 were females.

\section{Bacterial isolation}

The common organism isolated from the urine of all patients was E. coli. In diabetic patients, E. coli was found in 48 non-diabetic patients (Table I). Similarly, Candida spp. and Streptococcus faecalis were found to be 23.7 and $11.2 \%$ in diabetic patients and 37.5 and $20 \%$ in non-diabetic patients respectively (Table I). Pseudomonas aeruginosa and Pneumococcus were isolated only in non-diabetic patients, whereas mathicilline resistant S. aureus (MRSA), Proteus spp. and vancomycin resistant Enterococcus spp. were found only in diabetic patients (Table I).

\section{Determination of antimicrobial susceptibility}

We used disc diffusion method to determine the antibacterial susceptibility of isolated bacteriuria. From the results mentioned in Table II, it has been clearly found that E. coli exhibited maximum sensitivity against imipenem and meropenem (96\%) followed by amikacin that was $74 \%$. Whereas, E. coli exhibited antimicrobial resistance to all other antibacterial agents. We also found that E. coli did not show any kind of susceptibility to some anti- 
Table I

\begin{tabular}{|c|c|c|c|}
\hline \multirow[t]{2}{*}{ Bacteria found } & \multicolumn{2}{|c|}{$\begin{array}{c}\text { Number } \\
\text { (percentage) }\end{array}$} & \multirow[t]{2}{*}{$p$ value } \\
\hline & Diabetic & Non-diabetic & \\
\hline Escherichia coli & $\begin{array}{r}48 \\
(60)\end{array}$ & $\begin{array}{r}26 \\
(32.5)\end{array}$ & 0.007 \\
\hline Candida spp. & $\begin{array}{r}19 \\
(23.7)\end{array}$ & $\begin{array}{r}30 \\
(37.5)\end{array}$ & \\
\hline Streptococcus faecalis & $\begin{array}{r}9 \\
(11.2)\end{array}$ & $\begin{array}{r}16 \\
(20)\end{array}$ & \\
\hline Pseudomonas aeruginosa & 0 & $\begin{array}{r}5 \\
(6.2)\end{array}$ & \\
\hline Pneumococcus & 0 & $\begin{array}{r}2 \\
(2.5)\end{array}$ & \\
\hline $\begin{array}{l}\text { Methicillin-susceptible } \\
\text { Staphylococcus aureus }\end{array}$ & $\begin{array}{r}1 \\
(1.2)\end{array}$ & $\begin{array}{r}1 \\
(1.2)\end{array}$ & \\
\hline $\begin{array}{l}\text { Methicillin-resistance } \\
\text { Staphylococcus aureus }\end{array}$ & $\begin{array}{r}1 \\
(1.2)\end{array}$ & 0 & \\
\hline Proteus spp. & $\begin{array}{r}1 \\
(1.2)\end{array}$ & 0 & \\
\hline $\begin{array}{l}\text { Vancomycin resistant } \\
\text { Enterococcus spp. }\end{array}$ & $\begin{array}{r}1 \\
(1.2)\end{array}$ & 0 & \\
\hline
\end{tabular}

biotics namely ampicillin, amoxicillin, vancomycin and doxycycline. S. faecalis was susceptible to all antimicrobial agents that are mentioned in Table II. Vancomycin showed $88 \%$ sensitivity to $S$. faecalis while all other antibiotics found resistant to $S$. faecalis in greater ratio as shown in Table II. Imipenem, meropenem and amikacin exhibited over $80 \%$ sensitivity for $P$. aeruginosa and remaining antibiotics showed greater ratio of resistance to $P$. aeruginosa. Pneumococcus showed $100 \%$ sensitivity to imipenem, meropenem, amikacin, ciprofloxacin and levofloxacin while it showed resistance to other antibiotics used (Table II).

We found that mathicilline sensitive $S$. aureus (MSSA) exhibited its sensitivity $(100 \%)$ to clavulanic acid, cefepime, cefoperazone, imipenem, meropenem, amikacin, gentamycin, doxycycline, nitrofuantion and nalidixic acid while other antibiotics used were resistant to MSSA as shown in Table II. MRSA exhibited 100\% sensitivity to doxycycline and nitrofurantoin while all other antibiotics used were found resistant. Cefepime, cefoperazone, imipenem, meropenem and nitrofurantoin were found $100 \%$ sensitive to proteus spp. while remaining antibiotics used were found resistant. Nitrofurantion was the only antibiotic that was found be sensitive to vancomycin resistant Enterococcus spp. and remain-ing antibiotics used were found resistant (Table II).

Table II

\begin{tabular}{|c|c|c|c|c|c|c|c|c|c|}
\hline \multicolumn{10}{|c|}{ Antimicrobial sensitivity of UTI isolates } \\
\hline $\begin{array}{l}\text { Anti-microbial } \\
\text { agents }\end{array}$ & $\begin{array}{l}\text { Suscep- } \\
\text { tibility } \\
\text { pattern }\end{array}$ & E. coli & S. faecalis & P. aeruginosa & $\begin{array}{l}\text { Pneuтосос- } \\
\text { сиs }\end{array}$ & MSSA & MRSA & Proteus spp. & VRE spp. \\
\hline \multirow[t]{2}{*}{ Ampicillin } & S & - & $\begin{array}{r}10 \\
(40)\end{array}$ & - & - & 0 & 0 & - & 0 \\
\hline & $\mathrm{R}$ & - & $\begin{array}{r}15 \\
(60)\end{array}$ & - & - & $\begin{array}{r}2 \\
(100)\end{array}$ & $\begin{array}{r}1 \\
(100)\end{array}$ & - & $\begin{array}{r}1 \\
(100)\end{array}$ \\
\hline \multirow[t]{2}{*}{ Amoxicillin } & S & - & $\begin{array}{r}10 \\
(40)\end{array}$ & - & - & 0 & 0 & - & 0 \\
\hline & $\mathrm{R}$ & - & $\begin{array}{r}15 \\
(60)\end{array}$ & - & - & $\begin{array}{r}2 \\
(100)\end{array}$ & $\begin{array}{r}1 \\
(100)\end{array}$ & - & $\begin{array}{r}1 \\
(100)\end{array}$ \\
\hline \multirow[t]{2}{*}{ Clavulanic acid } & S & $\begin{array}{r}14 \\
(19)\end{array}$ & $\begin{array}{r}7 \\
(28)\end{array}$ & $\begin{array}{r}1 \\
(20)\end{array}$ & 0 & $\begin{array}{r}2 \\
(100)\end{array}$ & 0 & - & 0 \\
\hline & $\mathrm{R}$ & $\begin{array}{r}60 \\
(81)\end{array}$ & $\begin{array}{r}18 \\
(72)\end{array}$ & $\begin{array}{r}4 \\
(80)\end{array}$ & $\begin{array}{r}2 \\
(100)\end{array}$ & 0 & $\begin{array}{r}1 \\
(100)\end{array}$ & & $01(100)$ \\
\hline \multirow[t]{2}{*}{ Cefepime } & S & $\begin{array}{r}7 \\
(10)\end{array}$ & $\begin{array}{r}3 \\
(12)\end{array}$ & $\begin{array}{r}2 \\
(40)\end{array}$ & 0 & $\begin{array}{r}2 \\
(100)\end{array}$ & 0 & $\begin{array}{r}1 \\
(100)\end{array}$ & - \\
\hline & $\mathrm{R}$ & $\begin{array}{r}67 \\
(90)\end{array}$ & $\begin{array}{r}22 \\
(88)\end{array}$ & $\begin{array}{r}3 \\
(60)\end{array}$ & $\begin{array}{r}2 \\
(100)\end{array}$ & 0 & $\begin{array}{r}1 \\
(100)\end{array}$ & 0 & - \\
\hline \multirow[t]{2}{*}{ Cefoperazone } & S & $\begin{array}{r}7 \\
(10)\end{array}$ & $\begin{array}{r}3 \\
(12)\end{array}$ & $\begin{array}{r}2 \\
(40)\end{array}$ & 0 & $\begin{array}{r}2 \\
(100)\end{array}$ & 0 & $\begin{array}{r}1 \\
(100)\end{array}$ & - \\
\hline & $\mathrm{R}$ & $\begin{array}{r}67 \\
(90)\end{array}$ & $\begin{array}{r}22 \\
(88)\end{array}$ & $\begin{array}{r}3 \\
(60)\end{array}$ & $\begin{array}{r}2 \\
(100)\end{array}$ & 0 & $\begin{array}{r}1 \\
(100)\end{array}$ & 0 & - \\
\hline
\end{tabular}

Data are expressed as number (\% within the parenthesis) 
Table II

\section{Antimicrobial sensitivity of UTI isolates (Cont.)}

\begin{tabular}{|c|c|c|c|c|c|c|c|c|c|}
\hline $\begin{array}{l}\text { Anti-microbial } \\
\text { agents }\end{array}$ & $\begin{array}{c}\text { Suscep- } \\
\text { tibility } \\
\text { pattern }\end{array}$ & E. coli & S. faecalis & P. aeruginosa & $\begin{array}{l}\text { Pneuтосос- } \\
\text { сus }\end{array}$ & MSSA & MRSA & Proteus spp. & VRE spp. \\
\hline \multirow[t]{2}{*}{ Imipenem } & $\mathrm{S}$ & $\begin{array}{r}71 \\
(96)\end{array}$ & $\begin{array}{r}10 \\
(40)\end{array}$ & $4(80)$ & $\begin{array}{r}2 \\
(100)\end{array}$ & $\begin{array}{r}2 \\
(100)\end{array}$ & 0 & $\begin{array}{r}1 \\
(100)\end{array}$ & - \\
\hline & $\mathrm{R}$ & $\begin{array}{r}3 \\
(04)\end{array}$ & $\begin{array}{r}15 \\
(60)\end{array}$ & $\begin{array}{r}1 \\
(20)\end{array}$ & 0 & 0 & $\begin{array}{r}1 \\
(100)\end{array}$ & 0 & - \\
\hline \multirow[t]{2}{*}{ Meropenem } & $\mathrm{S}$ & $\begin{array}{r}71 \\
(96)\end{array}$ & $\begin{array}{r}10 \\
(40)\end{array}$ & $\begin{array}{r}4 \\
(80)\end{array}$ & $\begin{array}{r}2 \\
(100)\end{array}$ & $\begin{array}{r}2 \\
(100)\end{array}$ & 0 & $\begin{array}{r}1 \\
(100)\end{array}$ & - \\
\hline & $\mathrm{R}$ & $\begin{array}{r}3 \\
(04)\end{array}$ & $\begin{array}{r}15 \\
(60)\end{array}$ & $\begin{array}{r}1 \\
(20)\end{array}$ & 0 & 0 & $\begin{array}{r}1 \\
(100)\end{array}$ & 0 & - \\
\hline \multirow[t]{2}{*}{ Vancomycin } & $\mathrm{S}$ & - & $\begin{array}{r}22 \\
(88)\end{array}$ & - & - & $\begin{array}{r}2 \\
(100)\end{array}$ & - & - & 0 \\
\hline & $\mathrm{R}$ & - & $\begin{array}{r}3 \\
(12)\end{array}$ & - & - & 0 & - & - & $\begin{array}{r}1 \\
(100)\end{array}$ \\
\hline \multirow[t]{2}{*}{ Amikacin } & S & $\begin{array}{r}55 \\
(74)\end{array}$ & - & $\begin{array}{r}4 \\
(80)\end{array}$ & $\begin{array}{r}2 \\
(100)\end{array}$ & $\begin{array}{r}2 \\
(100)\end{array}$ & - & 0 & - \\
\hline & $\mathrm{R}$ & $\begin{array}{r}19 \\
(26)\end{array}$ & - & $\begin{array}{r}1 \\
(20)\end{array}$ & 0 & 0 & - & $\begin{array}{r}1 \\
(100)\end{array}$ & - \\
\hline \multirow[t]{2}{*}{ Gentamycin } & S & $\begin{array}{r}17 \\
(23)\end{array}$ & $\begin{array}{r}3 \\
(12)\end{array}$ & $\begin{array}{r}1 \\
(20)\end{array}$ & 0 & $\begin{array}{r}2 \\
(100)\end{array}$ & 0 & 0 & 0 \\
\hline & $\mathrm{R}$ & $\begin{array}{r}57 \\
(77)\end{array}$ & $\begin{array}{r}22 \\
(88)\end{array}$ & $\begin{array}{r}4 \\
(80)\end{array}$ & $\begin{array}{r}2 \\
(100)\end{array}$ & 0 & $\begin{array}{r}1 \\
(100)\end{array}$ & $\begin{array}{r}1 \\
(100)\end{array}$ & $\begin{array}{r}1 \\
(100)\end{array}$ \\
\hline \multirow[t]{2}{*}{ Doxycycline } & S & - & $\begin{array}{r}4 \\
(16)\end{array}$ & $\begin{array}{r}1 \\
(20)\end{array}$ & - & $\begin{array}{r}2 \\
(100)\end{array}$ & $\begin{array}{r}1 \\
(100)\end{array}$ & - & 0 \\
\hline & $\mathrm{R}$ & - & $\begin{array}{r}21 \\
(84)\end{array}$ & $\begin{array}{r}4 \\
(80)\end{array}$ & - & 0 & 0 & - & $\begin{array}{r}1 \\
(100)\end{array}$ \\
\hline \multirow[t]{2}{*}{ Ciprofloxacin } & $S$ & $\begin{array}{r}14 \\
(19)\end{array}$ & $\begin{array}{r}5 \\
(20)\end{array}$ & $\begin{array}{r}1 \\
(20)\end{array}$ & $\begin{array}{r}2 \\
(100)\end{array}$ & $\begin{array}{r}1 \\
(50)\end{array}$ & 0 & 0 & 0 \\
\hline & $\mathrm{R}$ & $\begin{array}{r}60 \\
(81)\end{array}$ & $\begin{array}{r}20 \\
(80)\end{array}$ & $\begin{array}{r}4 \\
(80)\end{array}$ & 0 & $\begin{array}{r}1 \\
(50)\end{array}$ & $\begin{array}{r}1 \\
(100)\end{array}$ & $\begin{array}{r}1 \\
(100)\end{array}$ & $\begin{array}{r}1 \\
(100)\end{array}$ \\
\hline \multirow[t]{2}{*}{ Levofloxacin } & S & $\begin{array}{r}14 \\
(19)\end{array}$ & $\begin{array}{r}5 \\
(20)\end{array}$ & $\begin{array}{r}1 \\
(20)\end{array}$ & $\begin{array}{r}2 \\
(100)\end{array}$ & $\begin{array}{r}1 \\
(50)\end{array}$ & 0 & 0 & 0 \\
\hline & $\mathrm{R}$ & $\begin{array}{r}60 \\
(81)\end{array}$ & $\begin{array}{r}20 \\
(80)\end{array}$ & $\begin{array}{r}4 \\
(80)\end{array}$ & 0 & $\begin{array}{r}1 \\
(50)\end{array}$ & $\begin{array}{r}1 \\
(100)\end{array}$ & $\begin{array}{r}1 \\
(100)\end{array}$ & $\begin{array}{r}1 \\
(100)\end{array}$ \\
\hline \multirow[t]{2}{*}{$\begin{array}{l}\text { Sulfamethoxa- } \\
\text { zole }\end{array}$} & $\mathrm{S}$ & $\begin{array}{r}11 \\
(15)\end{array}$ & $\begin{array}{r}10 \\
(40)\end{array}$ & $\begin{array}{r}2 \\
(40)\end{array}$ & - & $\begin{array}{r}1 \\
(50)\end{array}$ & 0 & - & - \\
\hline & $\mathrm{R}$ & $\begin{array}{r}63 \\
(85)\end{array}$ & $\begin{array}{r}15 \\
(60)\end{array}$ & $\begin{array}{r}3 \\
(60)\end{array}$ & - & $\begin{array}{r}1 \\
(50)\end{array}$ & $\begin{array}{r}1 \\
(100)\end{array}$ & - & - \\
\hline \multirow[t]{2}{*}{ Nitrofurantion } & S & $\begin{array}{r}11 \\
(15)\end{array}$ & $\begin{array}{r}7 \\
(28)\end{array}$ & $\begin{array}{r}1 \\
(20)\end{array}$ & 0 & $\begin{array}{r}2 \\
(100)\end{array}$ & $\begin{array}{r}1 \\
(100)\end{array}$ & $\begin{array}{r}1 \\
(100)\end{array}$ & $\begin{array}{r}1 \\
(100)\end{array}$ \\
\hline & $\mathrm{R}$ & $\begin{array}{r}63 \\
(85)\end{array}$ & $\begin{array}{r}18 \\
(72)\end{array}$ & $\begin{array}{r}4 \\
(80)\end{array}$ & $\begin{array}{r}2 \\
(100)\end{array}$ & 0 & 0 & 0 & 0 \\
\hline \multirow[t]{2}{*}{ Pipemedic acid } & $S$ & $\begin{array}{r}7 \\
(10)\end{array}$ & $\begin{array}{r}4 \\
(16)\end{array}$ & $\begin{array}{r}1 \\
(20)\end{array}$ & 0 & $\begin{array}{r}1 \\
(50)\end{array}$ & 0 & 0 & 0 \\
\hline & $\mathrm{R}$ & $\begin{array}{r}67 \\
(90)\end{array}$ & $\begin{array}{r}21 \\
(84)\end{array}$ & $\begin{array}{r}4 \\
(80)\end{array}$ & $\begin{array}{r}2 \\
(100)\end{array}$ & $\begin{array}{r}1 \\
(50)\end{array}$ & $\begin{array}{r}1 \\
(100)\end{array}$ & $\begin{array}{r}1 \\
(100)\end{array}$ & $\begin{array}{r}1 \\
(100)\end{array}$ \\
\hline \multirow[t]{2}{*}{ Nalidixic acid } & S & $\begin{array}{r}71 \\
(96)\end{array}$ & $\begin{array}{r}3 \\
(12)\end{array}$ & $\begin{array}{r}2 \\
(40)\end{array}$ & 0 & $\begin{array}{r}2 \\
(100)\end{array}$ & 0 & 0 & 0 \\
\hline & $\mathrm{R}$ & $\begin{array}{r}3 \\
(04)\end{array}$ & $\begin{array}{r}22 \\
(88)\end{array}$ & $\begin{array}{r}3 \\
(60)\end{array}$ & $\begin{array}{r}2 \\
(100)\end{array}$ & 0 & $\begin{array}{r}1 \\
(100)\end{array}$ & $\begin{array}{r}1 \\
(100)\end{array}$ & $\begin{array}{r}1 \\
(100)\end{array}$ \\
\hline
\end{tabular}

Data are expressed as number (\% within the parenthesis) 


\section{Discussion}

In the present study, we found that incidences of UTIs in diabetic patients are maximum than nondiabetics. Prevalence of UTIs were found more in female patients $(63.7 \%)$ than in male patients $(36.2 \%)$ which indicate that female patients (particularly diabetic) are more susceptible to UTIs as compared to male patients. The prevalence of UTIs among the study participants of present study was in accordance with already published reports. $\underline{12}$ $\underline{14}$ The prevalence of UTI was also described in renal transplant recipients..$\underline{15}$

Moreover, in present study, the most common uropathogen that was detected in the urine of all participants was E. coli. In diabetic patients, E. coli was detected in $60 \%$ patients, whereas in nondiabetic patients, it was detected in up to $32 \%$ patients. The increased prevalence of E. coli in UTIs indicates that $E$. coli is the leading and most significant isolate that is responsible to cause UTIs more significantly in diabetic patients especially females than non-diabetic patients.

\section{Conclusion}

Both diabetic and non-diabetic patients were susceptible to UTIs in which E. coli was found to be the most frequent uropathogen that exhibited its maximum susceptibility against tested antibacterial agents. Among the diabetic patient having UTIs, female diabetic patients were more susceptible to UTIs as compared to male diabetics which indicate that prevalence of UTIs in female diabetic patients is high as compared to that of male diabetic patients. Therefore, continued surveillance of prevalence of bacteriuria mandatory to ensure the appropriate diagnosis and recommendations for treatment of UTIs.

\section{References}

1. Akash MS, Rehman K, Chen S. Role of inflammatory mechanisms in pathogenesis of type 2 diabetes mellitus. J Cellular Biochem. 2013; 114: 525-31.

2. Akash MSH, Rehman K, Chen S. An overview of valuable scientific models for diabetes mellitus. Curr Diabetes Rev. 2013; 9: 286-93.

3. Akash MSH, Rehman K, Sun H, Chen S. Interleukin -1 receptor antagonist improves normoglycemia and insulin sensitivity in diabetic goto-kakizakirats. European J Pharmacol. 2013; 701: 87-95.
4. Tantry BA, Rahiman S. Antibacterial resistance and trend of urinary tract pathogens to commonly used antibiotics in Kashmir valley. West Indian Med J. 2012; 61: 703-07.

5. Gorter KJ, Hak E, Zuithoff NP, Hoepelman AI, Rutten GE. Risk of recurrent acute lower urinary tract infections and prescription pattern of antibiotics in women with and without diabetes in primary care. Fam Pract. 2010; 27: 379-85.

6. Patterson JE, Andriole VT. Bacterial urinary tract infections in diabetes. Infect Dis Clin North Am. 1997; 11: 735-50

7. Schneeberger C, Kazemier BM, Geerlings SE. Asymptomatic bacteriuria and urinary tract infections in special patient groups: Women with diabetes mellitus and pregnant women. Curr Opin Infect Dis. 2014; 27: 108-14

8. Shill MC, Huda NH, Moain FB, Karmakar UK. Prevalence of uropathogens in diabetic patients and their corresponding resistance pattern: Results of a survey conducted at diagnostic centers in Dhaka, Bangladesh. Oman Med J. 2010; 25: 282-85.

9. Jalali M, Shamsi M, Roozbehani N, Kabir K. Prevalence of urinary tract infection and some factors affected in pregnant women in Iran Karaj city 2013. Middle-East J Sci Res. 2014; 20: 781-85.

10. Kaspar L. [an absence of interaction of sulfametroltrimethoprim with insulin or sulphonylurea derivatives in diabetics (author's transl)]. Wiener klinische Wochenschrift. 1980; 92: 276-79.

11. Meiland R, Geerlings SE, Stolk RP, Netten PM, Schneeberger PM, Hoepelman AI. Asymptomatic bacteriuria in women with diabetes mellitus: Effect on renal function after 6 years of follow-up. Arch Intern Med. 2006; 166: 2222-27.

12. Ghenghesh KS, Elkateb E, Berbash N, Abdel Nada R, Ahmed SF, Rahouma A, et al. Uropathogens from diabetic patients in Libya: Virulence factors and phylogenetic groups of Escherichia coli isolates. J Med Microbiol. 2009; 58: 1006-14.

13. Ramana B, Chaudhury A. Antibiotic sensitivity pattern of Moraxella catarrhalis at a tertiary care hospital. Int J Pharm Life Sci. 2012; 3: 1805-06.

14. Yeshitela B, Gebre-Selassie S, Feleke Y. Asymptomatic bacteriuria and symptomatic urinary tract infections (uti) in patients with diabetes mellitus in Tikur Anbessa specialized university hospital, Addis Ababa, Ethiopia. Ethiopian M J. 2012; 50: 239 -49 .

15. Khan R, Roy C, Nowroz A, Nigar I, Saleh A. Urinary tract infection and their risk factors association in renal transplant recipients. Bangabandhu Sheikh Mujib Med Univ J. 2014; 7: 129-33. 